\title{
ON CONVEX AND STARLIKE FUNCTIONS IN A SECTOR
}

\section{NUNOKAWA and D. K. THOMAS}

(Received 5 Aug 1994; revised 10 Jan 1995)

\author{
Communicated by P. C. Fenton
}

\begin{abstract}
Let $f$ be analytic in $D=\{z:|z|<1\}$ with $f(0)=f^{\prime}(0)-1=0$. For $\gamma>0$, the largest $\alpha(\gamma)$ and $\beta(\gamma)$ are found such that

$$
1+\frac{z f^{\prime \prime}(z)}{f^{\prime}(z)} \prec\left(\frac{1+z}{1-z}\right)^{\alpha} \Longrightarrow \frac{z f^{\prime}(z)}{f(z)} \prec\left(\frac{1+z}{1-z}\right)^{\beta} \Longrightarrow \frac{f(z)}{z} \prec\left(\frac{1+z}{1-z}\right)^{\gamma}
$$

The results solve the inclusion problem for convex and starlike functions defined in a sector. 1991 Mathematics subject classification (Amer. Math. Soc.): $30 \mathrm{C} 45$.
\end{abstract}

\section{Introduction}

For $0 \leq \alpha<1$, let $K(\alpha)$ and $S t(\alpha)$ denote the classes of functions $f$ analytic in $D=\{z:|z|<1\}$ with $f(0)=f^{\prime}(0)-1=0$ which are convex and starlike of order $\alpha$; that is, which satisfy

$$
\operatorname{Re}\left(1+\frac{z f^{\prime \prime}(z)}{f^{\prime}(z)}\right)>\alpha
$$

and

$$
\operatorname{Re} \frac{z f^{\prime}(z)}{f(z)}>\alpha
$$

respectively.

In [2] and [7] MacGregor, Wilken and Feng determined the exact $\beta(\alpha)$ such that $K(\alpha) \subset S t(\beta)$ and in [1], Brickman et al found the exact $\gamma(\alpha)$ such that $f \in K(\alpha)$ implies $\operatorname{Re} f(z) / z>\gamma(\alpha)$, thus generalising Marx [3] and Strohhäcker's [6] classical

(c) 1996 Australian Mathematical Society 0263-6115/96 \$A2.00+0.00 
results in the case $\alpha=0$. By considering the extreme points of the closed convex hull of $S t(\alpha)$, it follows easily from the results in [1] that if $f \in S t(\alpha)$, then

$$
\operatorname{Re} \frac{f(z)}{z}>2^{-2(1-\alpha)} \quad \text { if } \quad \frac{1}{2} \leq \alpha<1,
$$

but that if $0 \leq \alpha<1 / 2$, then $\operatorname{Re} f(z) / z$ can become minus infinity.

In this paper, we solve the corresponding problems for the classes $C(\alpha)$ and $S^{*}(\alpha)$ of convex and starlike functions in a sector defined as follows.

Let $f$ be analytic in $D$ and $f(0)=f^{\prime}(0)-1=0$. Then for $0<\alpha \leq 1$ the classes $C(\alpha)$ and $S^{*}(\alpha)$ are defined by

$$
\left|\arg \left(1+\frac{z f^{\prime \prime}(z)}{f^{\prime}(z)}\right)\right|<\frac{\alpha \pi}{2},
$$

and

$$
\left|\arg \frac{z f^{\prime}(z)}{f(z)}\right|<\frac{\alpha \pi}{2}
$$

respectively. Our results are an immediate consequence of the following subordination theorem.

\section{Results}

THEOREM 1. Let $f$ be analytic in D with $f(0)=f^{\prime}(0)-1=0$ and $0<\beta \leq 1$. Then for $z \in D$,

$$
1+\frac{z f^{\prime \prime}(z)}{f^{\prime}(z)} \prec\left(\frac{1+z}{1-z}\right)^{\alpha(\beta)}
$$

implies

$$
\frac{z f^{\prime}(z)}{f(z)} \prec\left(\frac{1+z}{1-z}\right)^{\beta}
$$

where

$$
\alpha(\beta)=\frac{2}{\pi} \arctan \left[\tan \frac{\beta \pi}{2}+\frac{\beta}{(1+\beta)^{(1+\beta) / 2}(1-\beta)^{(1-\beta) / 2} \cos (\beta \pi / 2)}\right]
$$

Also for $\gamma>0$,

$$
\frac{z f^{\prime}(z)}{f(z)} \prec\left(\frac{1+z}{1-z}\right)^{\beta(\gamma)}
$$


implies

$$
\frac{f(z)}{z} \prec\left(\frac{1+z}{1-z}\right)^{\gamma}
$$

where

$$
\beta(\gamma)=\frac{2}{\pi} \arctan \gamma
$$

Furthermore $\alpha(\beta)$ is the largest number such that (1) holds and $\beta(\gamma)$ is the largest number such that (4) holds.

We shall use the following lemma (see for example [4]).

LEMMA. Let $F$ be analytic in $D$ and $G$ be analytic and univalent in $\bar{D}$, with $F(0)=G(0)$. If $F \nprec G$, then there is a point $z_{0} \in D$ and $\zeta_{0} \in \partial D$ such that $F\left(|z|<\left|z_{0}\right|\right) \subset G(D), F\left(z_{0}\right)=G\left(\zeta_{0}\right)$ and $z_{0} F^{\prime}\left(z_{0}\right)=m \zeta_{0} G^{\prime}\left(\zeta_{0}\right)$ for $m \geq 1$.

PROOF OF THEOREM 1. Write

$$
p(z)=\frac{z f^{\prime}(z)}{f(z)}
$$

so that $p$ is analytic in $D$ and $p(0)=1$. Thus we need to show that

$$
p(z)+\frac{z p^{\prime}(z)}{p(z)} \prec\left(\frac{1+z}{1-z}\right)^{\alpha}
$$

implies

$$
p(z) \prec\left(\frac{1+z}{1-z}\right)^{\beta}
$$

whenever $\alpha=\alpha(\beta)$.

Now let $h(z)=[(1+z) /(1-z)]^{\alpha}$ and $q(z)=[(1+z) /(1-z)]^{\beta}$. Then $|\arg h(z)|<$ $\alpha \pi / 2$ and $|\arg q(z)|<\beta \pi / 2$. Suppose that $p \nprec q$, then from the Lemma there is a point $z_{0} \in D$ and $\zeta_{0} \in \partial D$ such that $p\left(z_{0}\right)=q\left(\zeta_{0}\right)$ and $p\left(|z|<\left|z_{0}\right|\right) \subset q(D)$.

We next note that $p(z) \neq 0$ for $z \in D$, since otherwise we can write $p(z)=$ $\left(z-z_{1}\right)^{k} p_{1}(z)$ for some $k \geq 1$ and $p_{1}$ analytic in $D$, such that $p_{1}\left(z_{1}\right) \neq 0$. Then

$$
p(z)+\frac{z p^{\prime}(z)}{p(z)}=\frac{z p_{1}^{\prime}(z)}{p_{1}(z)}+\frac{k z}{z-z_{1}}+\left(z-z_{1}\right)^{k} p_{1}(z) \text {. }
$$

Thus choosing $z \rightarrow z_{1}$, suitably the argument of the right-hand side of (6) can take any value between 0 and $2 \pi$, which contradicts the hypotheses of the Theorem. 
Since $p\left(z_{0}\right)=q\left(\zeta_{0}\right) \neq 0$, it follows that $\zeta_{0} \neq \pm 1$. Thus we can write $r i=$ $\left(1+\zeta_{0}\right) /\left(1-\zeta_{0}\right)$ for $r \neq 0$. Then the Lemma gives

$$
\begin{aligned}
p\left(z_{0}\right)+\frac{z_{0} p^{\prime}\left(z_{0}\right)}{p\left(z_{0}\right)} & =q\left(\zeta_{0}\right)+\frac{m \zeta_{0} q^{\prime}\left(\zeta_{0}\right)}{q\left(\zeta_{0}\right)} \\
& =(r i)^{\beta}+\frac{m \beta\left(1+r^{2}\right) i}{2 r} .
\end{aligned}
$$

Next assume that $r>0$. (If $r<0$, the proof is similar.) Since $m \geq 1$, an elementary argument shows that

$$
\begin{aligned}
\arg \left(p\left(z_{0}\right)+\frac{z_{0} p^{\prime}\left(z_{0}\right)}{p\left(z_{0}\right)}\right) & =\arctan \left[\tan \frac{\beta \pi}{2}+\frac{m \beta\left(1+r^{2}\right)}{2 r^{\beta+1} \cos (\beta \pi / 2)}\right] \\
& \geq \arctan \left[\tan \frac{\beta \pi}{2}+\frac{\beta\left(1+r^{2}\right)}{2 r^{\beta+1} \cos (\beta \pi / 2)}\right] \\
& \geq \arctan \left[\tan \frac{\beta \pi}{2}+\frac{\beta}{(1+\beta)^{(1+\beta) / 2}(1-\beta)^{(1-\beta) / 2} \cos (\beta \pi / 2)}\right] \\
& =\alpha(\beta) \pi / 2 .
\end{aligned}
$$

Hence

$$
\frac{\alpha(\beta) \pi}{2} \leq \arg \left(p\left(z_{0}\right)+\frac{z_{0} p^{\prime}\left(z_{0}\right)}{p\left(z_{0}\right)}\right) \leq \frac{\pi}{2},
$$

which contradicts the fact that $|\arg h(z)|<\alpha(\beta) \pi / 2$ provided that (2) holds.

To show that (2) is exact, let

$$
p(z)=\left(\frac{1+z}{1-z}\right)^{\beta} .
$$

Then from the minimum principle for harmonic functions, it follows that

$$
\inf _{|z|<1} \arg \left(p(z)+\frac{z p^{\prime}(z)}{p(z)}\right)
$$

is attained at some point $z=e^{i \theta}$ for $0<\theta<2 \pi$. Thus

$$
p(z)+\frac{z p^{\prime}(z)}{p(z)}=\left(\frac{\sin \theta}{1-\cos \theta}\right)^{\beta} e^{\beta \pi i / 2}+\frac{i \beta}{\sin \theta},
$$

and so writing $t=\cos \theta$, we obtain

$$
\arg \left(p(z)+\frac{z p^{\prime}(z)}{p(z)}\right)=\arctan \left[\tan \frac{\beta \pi}{2}+\frac{\beta}{(1+t)^{(1+\beta) / 2}(1-t)^{(1-\beta) / 2} \cos (\beta \pi / 2)}\right]
$$


and an elementary calculation shows that the minimum of this expression is attained when $t=\beta$.

We next show that (3) implies (4). Write $p(z)=f(z) / z$, so that again $p$ is analytic in $D$ and $p(0)=1$. Thus this time we need to show that

$$
1+\frac{z p^{\prime}(z)}{p(z)} \prec\left(\frac{1+z}{1-z}\right)^{\beta(\gamma)}
$$

implies

$$
p(z) \prec\left(\frac{1+z}{1-z}\right)^{\gamma}
$$

whenever (5) holds.

As before, let $h(z)=[(1+z) /(1-z)]^{\beta}$ and $q(z)=[(1+z) /(1-z)]^{\gamma}$ and assume that $p \nprec q$. Then there exists $z_{0} \in D$ and $\zeta_{0} \in \partial D$ such that $p\left(z_{0}\right)=q\left(\zeta_{0}\right)$ and $p\left(|z|<\left|z_{0}\right|\right) \subset q(D)$. Since $p\left(z_{0}\right)=q\left(\zeta_{0}\right) \neq 0$, the Lemma gives

$$
1+\frac{z_{0} p^{\prime}\left(z_{0}\right)}{p\left(z_{0}\right)}=1+\frac{m \gamma\left(1+r^{2}\right) i}{2 r}
$$

The result now follows using the same arguments as before.

To show that $\beta(\gamma)$ is the largest number such that (4) holds we again let $p(z)=$ $[(1+z) /(1-z)]^{\beta}$ and $z=e^{i \theta}$. Then

$$
\arg \left(1+\frac{z p^{\prime}(z)}{p(z)}\right)=\arctan \left[\frac{\beta}{\sin \theta}\right],
$$

which has a minimum when $\theta=\pi / 2$.

The following is an immediate consequence of Theorem 1.

COROLLARY. For $\gamma>0$,

$$
1+\frac{z f^{\prime \prime}(z)}{f^{\prime}(z)} \prec\left(\frac{1+z}{1-z}\right)^{\alpha(\gamma)}
$$

implies

$$
\frac{f(z)}{z} \prec\left(\frac{1+z}{1-z}\right)^{\gamma}
$$

where

$\alpha(\gamma)=\frac{2}{\pi} \arctan \left[\tan \frac{\beta(\gamma) \pi}{2}+\frac{\beta(\gamma)}{(1+\beta(\gamma))^{(1+\beta(\gamma)) / 2}(1-\beta(\gamma))^{(1-\beta(\gamma)) / 2} \cos (\beta(\gamma) \pi / 2)}\right]$

and $\beta(\gamma)$ is given by (5). Also $\alpha(\gamma)$ is the largest number such that (7) holds. 
For $\alpha>0$, denote by $R(\alpha)$ the class of functions $f$ analytic in $D$ with $f(0)=$ $f^{\prime}(0)-1=0$, satisfying

$$
\left|\arg \frac{f(z)}{z}\right|<\frac{\alpha \pi}{2},
$$

for $z \in D$. We now restate the results in terms of $C(\alpha), S^{*}(\alpha)$ and $R(\alpha)$ as follows.

THEOREM 2. For $\gamma>0$,

$$
C(\alpha(\beta(\gamma))) \subset S^{*}(\beta(\gamma)) \subset R(\gamma),
$$

where $\alpha(\beta)$ and $\beta(\gamma)$ are given by (2) and (5) respectively. Furthermore, $\alpha(\beta(\gamma))$ and $\beta(\gamma)$ are the largest numbers such that the inclusion holds.

\section{References}

[1] L. Brickman, D. J. Hallenbeck, T. M. Macgregor and D. R. Wilken, 'Convex hulls and extreme points of families of starlike and convex mappings', Trans. Amer. Math. Soc. 185 (1973), 413-428.

[2] T. H. Macgregor, 'A subordination for convex functions of order $\alpha$ ', J. London. Math. Soc. 9 (1975), $530-536$.

[3] A. Marx, 'Untersuchungen über schlicht Abbildungen,', Math. Ann. 107 (1932/33), $40-67$.

[4] S. S. Miller and P. T. Mocanu, 'Differential subordinations and univalent functions', Michigan Math. J. 28 (1981), 157-171.

[5] R. M. Robinson, 'Univalent majorants', Trans. Amer. Math. Soc. 61 (1947), 1-35.

[6] E. Strohhäcker, 'Beiträge zur Theorie der schlichten Funktionen', Math. Z. 37 (1933), 356-380.

[7] D. R. Wilken and J. Feng, 'A remark on convex and starlike functions', J. Lond. Math. Soc. 21 (1980), 287-290.

Department of Mathematics

Faculty of Education

Gunma University

Maebashi, Gunma 371

Japan
Department of Mathematics

University of Wales

Swansea SA2 8PP

UK

e-mail: d.k.thomas@swansea.ac.uk 